\title{
The genome sequence of the sycamore, Acronicta aceris
}

\section{(Linnaeus, 1758) [version 1; peer review: 2 approved]}

Douglas Boyes ${ }^{1+}$, Liam M. Crowley (iD2, Peter W.H. Holland (iD2, University of Oxford and Wytham Woods Genome Acquisition Lab, Darwin Tree of Life Barcoding collective, Wellcome Sanger Institute Tree of Life programme, Wellcome Sanger Institute Scientific Operations: DNA Pipelines collective, Tree of Life Core Informatics collective, Darwin Tree of Life Consortium

${ }^{1}$ UK Centre for Ecology \& Hydrology, Wallingford, UK

${ }^{2}$ Department of Zoology, University of Oxford, Oxford, UK

+ Deceased author

V1 First published: 26 Nov 2021, 6:326

https://doi.org/10.12688/wellcomeopenres.17354.1

Latest published: 26 Nov 2021, 6:326

https://doi.org/10.12688/wellcomeopenres.17354.1

\section{Abstract}

We present a genome assembly from an individual female Acronicta aceris (the sycamore; Arthropoda; Insecta; Lepidoptera; Noctuidae). The genome sequence is 466 megabases in span. The complete assembly is scaffolded into 32 chromosomal pseudomolecules, with the $\mathrm{W}$ and $\mathrm{Z}$ sex chromosome assembled.

Keywords

Acronicta aceris, the sycamore, genome sequence, chromosomal, Lepidoptera

This article is included in the Tree of Life gateway.

\section{Open Peer Review \\ Approval Status \\ 2 \\ version 1 \\ 26 Nov 2021

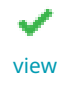 \\ view \\ 1. Kumar Saurabh Singh iD, Wageningen University, Wageningen, The Netherlands Utrecht University, Utrecht, The Netherlands University of Exeter, Cornwall Campus, Cornwall, UK \\ 2. Appolinaire Djikeng , University of Edinburgh, Edinburgh, UK}

Any reports and responses or comments on the article can be found at the end of the article. 
Corresponding author: Darwin Tree of Life Consortium (mark.blaxter@sanger.ac.uk)

Author roles: Boyes D: Investigation, Resources; Crowley LM: Writing - Original Draft Preparation, Writing - Review \& Editing; Holland PWH: Supervision, Writing - Original Draft Preparation, Writing - Review \& Editing;

Competing interests: No competing interests were disclosed.

Grant information: This work was supported by the Wellcome Trust through core funding to the Wellcome Sanger Institute (206194) and the Darwin Tree of Life Discretionary Award (218328).

The funders had no role in study design, data collection and analysis, decision to publish, or preparation of the manuscript.

Copyright: (c) 2021 Boyes D et al. This is an open access article distributed under the terms of the Creative Commons Attribution License, which permits unrestricted use, distribution, and reproduction in any medium, provided the original work is properly cited.

How to cite this article: Boyes D, Crowley LM, Holland PWH et al. The genome sequence of the sycamore, Acronicta aceris (Linnaeus, 1758) [version 1; peer review: 2 approved] Wellcome Open Research 2021, 6:326 https://doi.org/10.12688/wellcomeopenres.17354.1

First published: 26 Nov 2021, 6:326 https://doi.org/10.12688/wellcomeopenres.17354.1 


\section{Species taxonomy}

Eukaryota; Metazoa; Ecdysozoa; Arthropoda; Hexapoda; Insecta; Pterygota; Neoptera; Endopterygota; Lepidoptera; Glossata; Ditrysia; Noctuoidea; Noctuidae; Acronictinae; Acronicta; Acronicta aceris (Linnaeus, 1758) (NCBI:txid987859).

\section{Background}

Acronicta aceris (sycamore moth) is a widely distributed noctuid moth found in Europe, Morocco and western regions of Asia. In the UK it is locally common in southeast and central England, with a flight season from June to August. Forewing colouration of the moth varies from silvery to dark grey, with variation in ground colour likely controlled by alleles at a single locus (Majerus, 1986). The larvae of A. aceris are amongst the most colourful and flamboyant of all Lepidoptera caterpillars, bearing yellow and orange hairs arranged in striking 'punk' tufts along the body. As the common name suggests, the larvae feed on the leaves of sycamore (Acer pseudoplatanus), other maples (Acer sp.) and, particularly in urban and suburban areas, horse chestnut (Aesculus hippocastanum). Larvae are active from July to September and overwintering occurs as a pupa in a double-layered cocoon in bark crevices or leaf litter. It is known to occasionally overwinter as a pupa through two winters before eclosing as an imago (Waring et al., 2003).

\section{Genome sequence report}

The genome was sequenced from one female $A$. berbera (Figure 1) collected from Wytham Woods, Oxfordshire (biological vice-county: Berkshire), UK (latitude 51.772, longitude -1.338). A total of 39-fold coverage in Pacific Biosciences singlemolecule long reads and 99-fold coverage in 10X Genomics read clouds were generated. Primary assembly contigs were scaffolded with chromosome conformation Hi-C data. Manual assembly curation corrected $14 \mathrm{missing} /$ misjoins, reducing the scaffold number by $20.00 \%$ and increasing the scaffold N50 by $4.33 \%$.

The final assembly has a total length of $466 \mathrm{Mb}$ in 32 sequence scaffolds with a scaffold N50 of $16.1 \mathrm{Mb}$ (Table 1). The complete assembly sequence was assigned to 32 chromosomal-level scaffolds, representing 30 autosomes (numbered by sequence length), and the $\mathrm{W}$ and $\mathrm{Z}$ sex chromosome (Figure 2-Figure 5; Table 2). The assembly has a BUSCO v5.1.2 (Manni et al., 2021) completeness of $99.0 \%$ (single $98.5 \%$, duplicated $0.5 \%$ ) using the lepidoptera_odb10 reference set. While not fully phased, the assembly deposited is of one haplotype. Contigs corresponding to the second haplotype have also been deposited.

\section{Methods}

Sample acquisition and DNA extraction

A single female A. aceris (ilAcrAcer1) was collected from Wytham Woods, Oxfordshire (biological vice-county: Berkshire), UK (latitude 51.772, longitude -1.338) by Douglas Boyes, UKCEH, using a light trap. The sample was identified by the same individual, and preserved on dry ice.

DNA was extracted at the Tree of Life laboratory, Wellcome Sanger Institute. The ilAcrAcer1 sample was weighed and dissected on dry ice with tissue set aside for Hi-C sequencing. Abdomen tissue was cryogenically disrupted to a fine powder using a Covaris cryoPREP Automated Dry Pulveriser, receiving multiple impacts. Fragment size analysis of 0.01-0.5 ng of DNA was then performed using an Agilent FemtoPulse. High molecular weight (HMW) DNA was extracted using the Qiagen MagAttract HMW DNA extraction kit. Low molecular weight DNA was removed from a 200-ng aliquot of extracted DNA using 0.8X AMpure XP purification kit prior to $10 \mathrm{X}$ Chromium sequencing; a minimum of $50 \mathrm{ng}$ DNA was submitted for

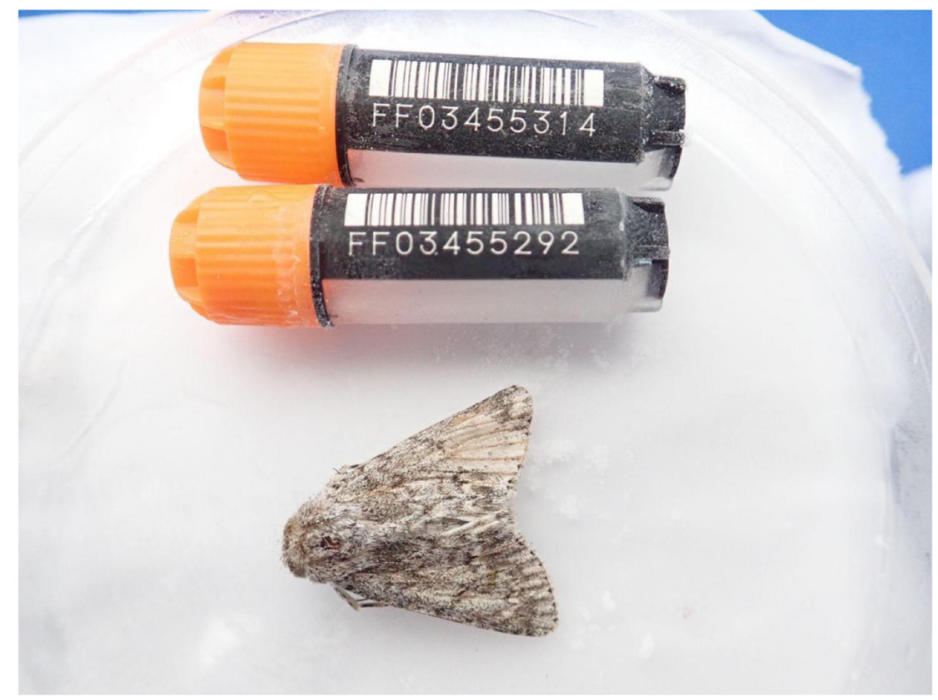

Figure 1. Image of the ilAcrAcer1 specimen captured prior to preservation and processing. Specimen shown next to FluidX storage tube, $43.9 \mathrm{~mm}$ in length. 
Table 1. Genome data for Acronicta aceris, ilAcrAcer1.1.

\begin{tabular}{|c|c|}
\hline \multicolumn{2}{|l|}{ Project accession data } \\
\hline Assembly identifier & ilAcrAcer1.1 \\
\hline Species & Acronicta aceris \\
\hline Specimen & ilAcrAcer1 \\
\hline NCBI taxonomy ID & NCBI:txid987859 \\
\hline BioProject & PRJEB45197 \\
\hline BioSample ID & SAMEA7701532 \\
\hline Isolate information & $\begin{array}{l}\text { Female, abdomen (genome assembly), head/ } \\
\text { thorax }(\mathrm{Hi}-\mathrm{C})\end{array}$ \\
\hline \multicolumn{2}{|l|}{ Raw data accessions } \\
\hline PacificBiosciences SEQUEL II & ERR6406216 \\
\hline 10X Genomics Illumina & ERR6054961-ERR6054964 \\
\hline Hi-C Illumina & ERR6054960 \\
\hline \multicolumn{2}{|l|}{ Genome assembly } \\
\hline Assembly accession & GCA_910591435.1 \\
\hline Accession of alternate haplotype & GCA_910591495.1 \\
\hline Span (Mb) & 466 \\
\hline Number of contigs & 45 \\
\hline Contig N50 length (Mb) & 15.4 \\
\hline Number of scaffolds & 32 \\
\hline Scaffold N50 length (Mb) & 16.1 \\
\hline Longest scaffold (Mb) & 19.0 \\
\hline BUSCO* genome score & C:99.0\%[S:98.5\%,D:0.5\%],F:0.1\%,M:0.9\%,n:5286 \\
\hline
\end{tabular}

$10 \mathrm{X}$ sequencing. HMW DNA was sheared into an average fragment size between 12-20 kb in a Megaruptor 3 system with speed setting 30. Sheared DNA was purified by solid-phase reversible immobilisation using AMPure PB beads with a $1.8 \mathrm{X}$ ratio of beads to sample to remove the shorter fragments and concentrate the DNA sample. The concentration of the sheared and purified DNA was assessed using a Nanodrop spectrophotometer and Qubit Fluorometer and Qubit dsDNA High Sensitivity Assay kit. Fragment size distribution was evaluated by running the sample on the FemtoPulse system.

\section{Sequencing}

Pacific Biosciences HiFi circular consensus and 10X Genomics read cloud DNA sequencing libraries were constructed according to the manufacturers' instructions. Sequencing was performed by the Scientific Operations core at the WSI on Pacific Biosciences SEQUEL II and Illumina HiSeq X instruments. $\mathrm{Hi}-\mathrm{C}$ data were generated from abdomen tissue using the Arima v2 Hi-C kit and sequenced on an Illumina NovaSeq $6000 \mathrm{X}$ instrument.

\section{Genome assembly}

Assembly was carried out with Hifiasm (Cheng et al., 2021); haplotypic duplication was identified and removed with purge_dups (Guan et al., 2020). One round of polishing was performed by aligning $10 \mathrm{X}$ Genomics read data to the assembly with longranger align, calling variants with freebayes (Garrison \& Marth, 2012). The assembly was then scaffolded with Hi-C data (Rao et al., 2014) using SALSA2 (Ghurye et al., 2019). The assembly was checked for contamination and corrected using 


\section{Scaffold statistics}

BUSCO lepidoptera_odb10 (5286)

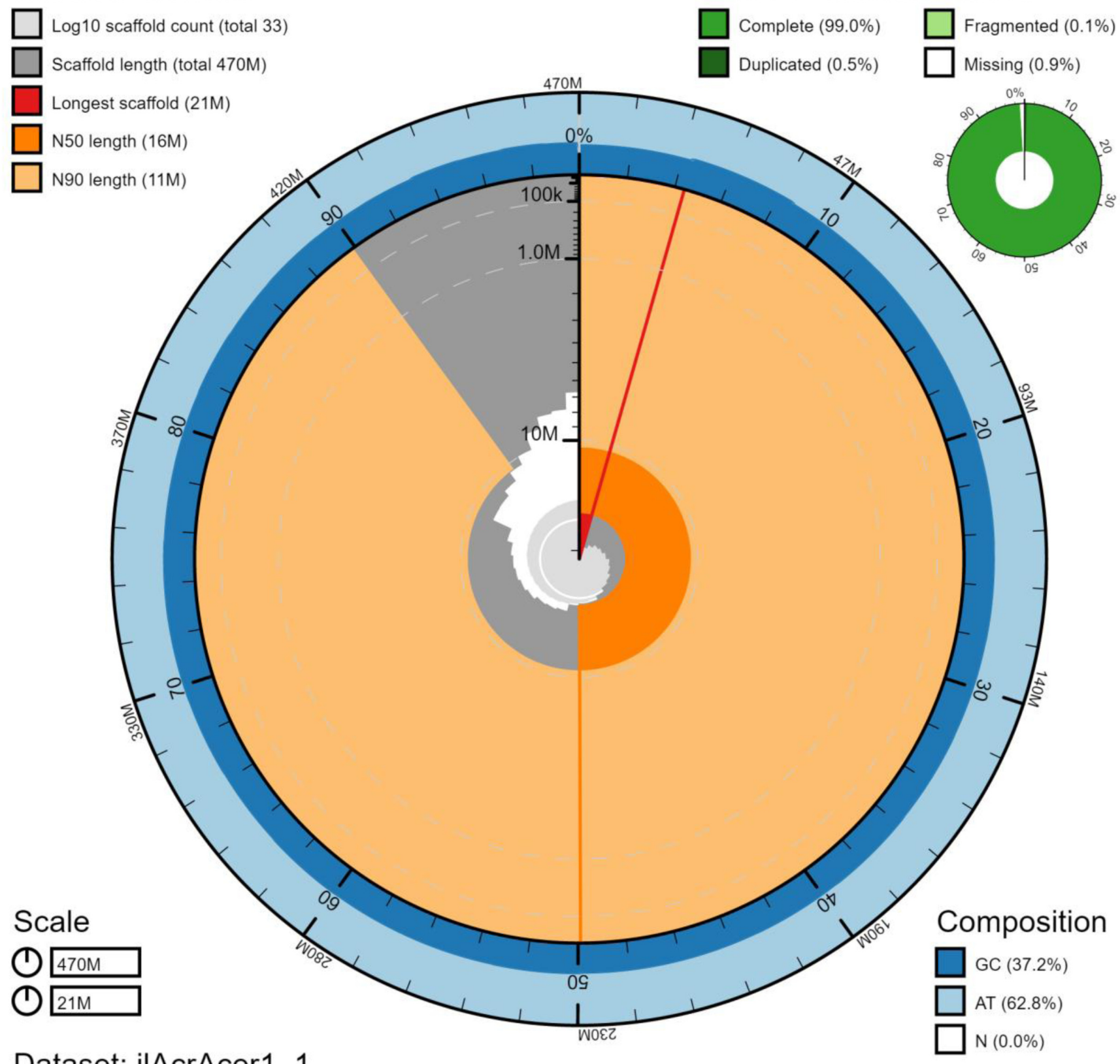

\section{Dataset: ilAcrAcer1_1}

Figure 2. Genome assembly of Acronicta aceris, ilAcrAcer1.1: metrics. The BlobToolKit Snailplot shows N50 metrics and BUSCO gene completeness. The main plot is divided into 1,000 size-ordered bins around the circumference with each bin representing $0.1 \%$ of the $466,384,436$ bp assembly. The distribution of chromosome lengths is shown in dark grey with the plot radius scaled to the longest chromosome present in the assembly (20,910,575 bp, shown in red). Orange and pale-orange arcs show the N50 and N90 chromosome lengths $(16,061,144$ and 10,539,460 bp), respectively. The pale grey spiral shows the cumulative chromosome count on a log scale with white scale lines showing successive orders of magnitude. The blue and pale-blue area around the outside of the plot shows the distribution of GC, AT and N percentages in the same bins as the inner plot. A summary of complete, fragmented, duplicated and missing BUSCO genes in the lepidoptera_odb10 set is shown in the top right. An interactive version of this figure is available at https://blobtoolkit.genomehubs. org/view/ilAcrAcer1.1/dataset/ilAcrAcer1_1/snail.

the gEVAL system (Chow et al., 2016) as described previously (Howe et al., 2021). Manual curation (Howe et al., 2021) was performed using gEVAL, HiGlass (Kerpedjiev et al., 2018) and Pretext. The mitochondrial genome was assembled using 


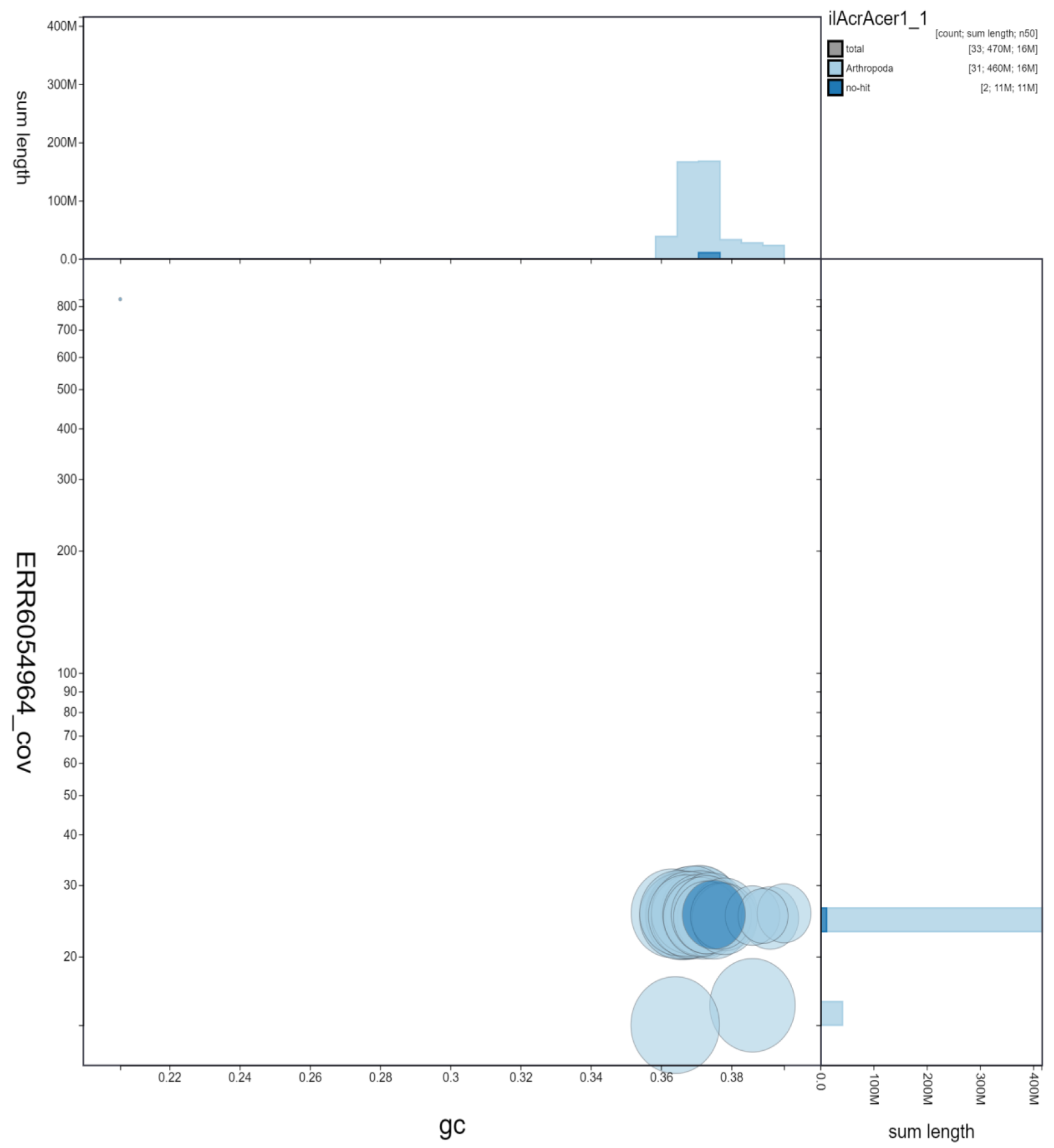

Figure 3. Genome assembly of Acronicta aceris, ilAcrAcer1.1: GC coverage. BlobToolKit GC-coverage plot. Scaffolds are coloured by phylum. Circles are sized in proportion to scaffold length. Histograms show the distribution of scaffold length sum along each axis. An interactive version of this figure is available at https://blobtoolkit.genomehubs.org/view/ilAcrAcer1.1/dataset/ilAcrAcer1_1/blob.

MitoHiFi (Uliano-Silva et al., 2021). The genome was analysed and BUSCO scores generated within the BlobToolKit environment (Challis et al., 2020). Table 3 contains a list of all software tool versions used, where appropriate.

\section{Ethics/compliance issues}

The materials that have contributed to this genome note have been supplied by a Darwin Tree of Life Partner. The submission of materials by a Darwin Tree of Life Partner is subject to the 


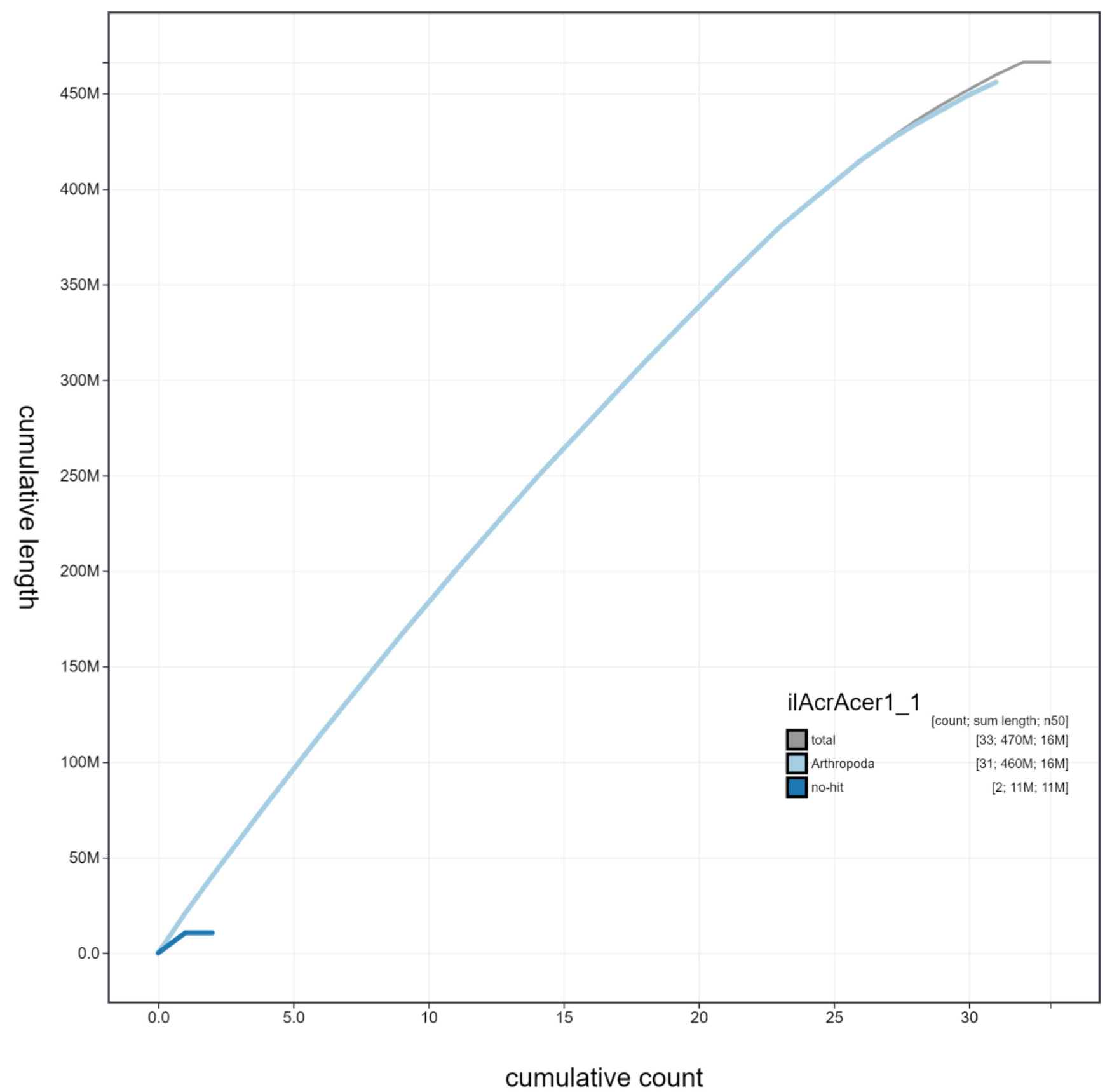

Figure 4. Genome assembly of Acronicta aceris, ilAcrAcer1.1: cumulative sequence. BlobToolKit cumulative sequence plot. The grey line shows cumulative length for all scaffolds. Coloured lines show cumulative lengths of scaffolds assigned to each phylum using the buscogenes taxrule. An interactive version of this figure is available at https://blobtoolkit.genomehubs.org/view/ilAcrAcer1.1/dataset/ ilAcrAcer1_1/cumulative.

Darwin Tree of Life Project Sampling Code of Practice. By agreeing with and signing up to the Sampling Code of Practice, the Darwin Tree of Life Partner agrees they will meet the legal and ethical requirements and standards set out within this document in respect of all samples acquired for, and supplied to, the Darwin Tree of Life Project. Each transfer of samples is further undertaken according to a Research Collaboration Agreement or Material Transfer Agreement entered into by the Darwin Tree of Life Partner, Genome Research Limited (operating as the Wellcome Sanger Institute), and in some circumstances other Darwin Tree of Life collaborators. 


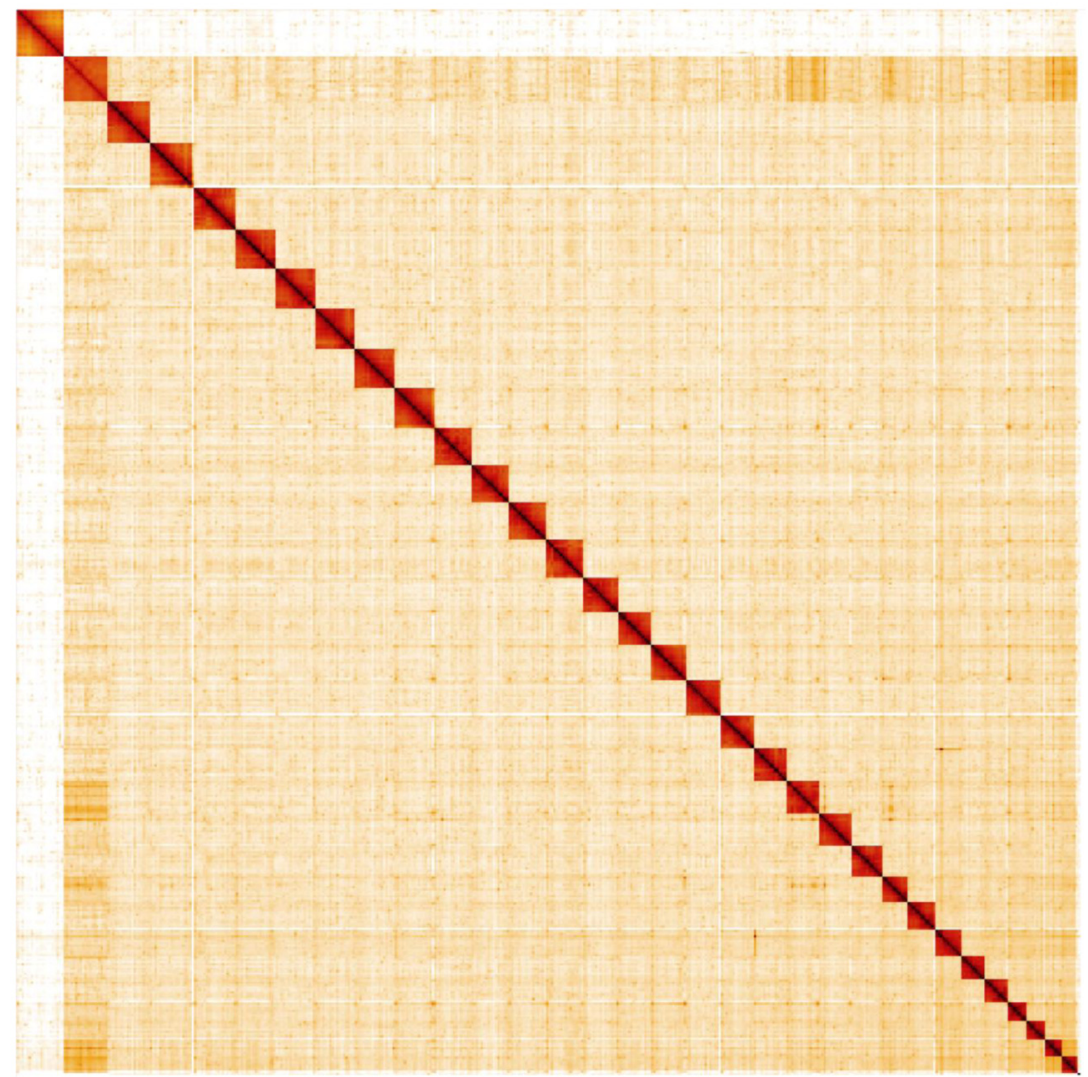

Figure 5. Genome assembly of Acronicta aceris, ilAcrAcer1.1: Hi-C contact map. Hi-C contact map of the ilAcrAcer1.1 assembly, visualised in HiGlass.

\begin{tabular}{|c|c|c|c|}
\hline $\begin{array}{l}\text { Table 2. Chromosomal pseudomolecules in the } \\
\text { genome assembly of Acronicta aceris, ilAcrAcer1.1. }\end{array}$ \\
\hline INSDC accession & Chromosome & Size (Mb) & GC\% \\
\hline OU342758.1 & 1 & 18.99 & 36.8 \\
\hline OU342759.1 & 2 & 18.58 & 37.1 \\
\hline OU342760.1 & 3 & 18.34 & 37.1 \\
\hline OU342761.1 & 4 & 18.02 & 36.9 \\
\hline OU342762.1 & 5 & 17.67 & 36.6 \\
\hline OU342763.1 & 6 & 17.51 & 36.3 \\
\hline OU342764.1 & 7 & 17.20 & 36.7 \\
\hline OU342765.1 & 8 & 16.99 & 37.2 \\
\hline OU342766.1 & 9 & 16.64 & 36.7 \\
\hline OU342767.1 & 10 & 16.27 & 36.5 \\
\hline OU342768.1 & 11 & 16.24 & 36.5 \\
\hline OU342769.1 & 12 & 16.06 & 37.1 \\
\hline OU342770.1 & 13 & 15.39 & 36.8 \\
\hline OU342771.1 & 14 & 15.23 & 36.8 \\
\hline OU342772.1 & 15 & 15.11 & 37.2 \\
\hline OU342773.1 & 16 & 14.79 & 36.7 \\
\hline
\end{tabular}

\begin{tabular}{|c|c|c|c|}
\hline INSDC accession & Chromosome & Size (Mb) & GC\% \\
\hline OU342774.1 & 17 & 14.58 & 37.1 \\
\hline OU342775.1 & 18 & 14.39 & 37.4 \\
\hline OU342776.1 & 19 & 14.24 & 37.5 \\
\hline OU342777.1 & 20 & 14.04 & 37.1 \\
\hline OU342778.1 & 21 & 13.53 & 37.3 \\
\hline OU342779.1 & 22 & 11.82 & 37.8 \\
\hline OU342780.1 & 23 & 11.79 & 37.3 \\
\hline OU342781.1 & 24 & 11.31 & 37.8 \\
\hline OU342782.1 & 25 & 10.54 & 37.5 \\
\hline OU342783.1 & 26 & 9.86 & 37.7 \\
\hline OU342784.1 & 27 & 8.67 & 39.1 \\
\hline OU342785.1 & 28 & 7.85 & 38.6 \\
\hline OU342786.1 & 29 & 7.70 & 39.5 \\
\hline OU342787.1 & 30 & 6.64 & 38.9 \\
\hline OU342757.1 & W & 19.46 & 38.6 \\
\hline OU342756.1 & Z & 20.91 & 36.4 \\
\hline OU342788.1 & MT & 0.02 & 20.6 \\
\hline
\end{tabular}


Table 3. Software tools used.

\begin{tabular}{|l|l|l|}
\hline Software tool & Version & Source \\
\hline Hifiasm & 0.14 & Cheng et al., 2021 \\
\hline purge_dups & 1.2 .3 & Guan et al., 2020 \\
\hline SALSA2 & 2.2 & Ghurye et al., 2019 \\
\hline longranger align & 2.2 .2 & $\begin{array}{l}\text { https://support.10xgenomics.com/genome-exome/ } \\
\text { software/pipelines/latest/advanced/other-pipelines }\end{array}$ \\
\hline freebayes & $1.3 .1-17$-gaa2ace8 & Garrison \& Marth, 2012 \\
\hline MitoHiFi & 3.0 & Uliano-Silva et al., 2021 \\
\hline gEVAL & N/A & Chow et al., 2016 \\
\hline HiGlass & 1.11 .6 & Kerpedjiev et al., 2018 \\
\hline PretextView & $0.2 . x$ & https://github.com/wtsi-hpag/PretextView \\
\hline BlobToolKit & 2.6 .2 & Challis et al., 2020 \\
\hline
\end{tabular}

\section{Data availability}

European Nucleotide Archive: Acronicta aceris (the sycamore). Accession number PRJEB45197; https://identifiers.org/ena.embl/ PRJEB45197.

The genome sequence is released openly for reuse. The $A$. aceris genome sequencing initiative is part of the Darwin Tree of Life (DToL) project. All raw sequence data and the assembly have been deposited in INSDC databases. The genome will be annotated and presented through the Ensembl pipeline at the European Bioinformatics Institute. Raw data and assembly accession identifiers are reported in Table 1.

\section{Author information}

Members of the University of Oxford and Wytham Woods Genome Acquisition Lab are listed here: https://doi.org/10.5281/ zenodo.4789929.
Members of the Darwin Tree of Life Barcoding collective are listed here: https://doi.org/10.5281/zenodo.4893704.

Members of the Wellcome Sanger Institute Tree of Life programme collective are listed here: https://doi.org/10.5281/ zenodo.5377053.

Members of Wellcome Sanger Institute Scientific Operations: DNA Pipelines collective are listed here: https://doi.org/10.5281/ zenodo.4790456.

Members of the Tree of Life Core Informatics collective are listed here: https://doi.org/10.5281/zenodo.5013542.

Members of the Darwin Tree of Life Consortium are listed here: https://doi.org/10.5281/zenodo.4783559.
Challis R, Richards E, Rajan J, et al.: BlobToolKit - Interactive Quality Assessment of Genome Assemblies. G3 (Bethesda). 2020; 10(4): 1361-74. PubMed Abstract | Publisher Full Text | Free Full Text

Cheng $\mathrm{H}$, Concepcion GT, Feng X, et al.: Haplotype-Resolved de Novo Assembly Using Phased Assembly Graphs with Hifiasm. Nat Methods. 2021; 18(2): 170-75.

PubMed Abstract | Publisher Full Text | Free Full Text

Chow W, Brugger K, Caccamo M, et al.: gEVAL - a web-based browser for evaluating genome assemblies. Bioinformatics. 2016; 32(16): 2508-10. PubMed Abstract | Publisher Full Text | Free Full Text

Garrison E, Marth G: Haplotype-Based Variant Detection from Short-Read Sequencing. arXiv: 1207.3907. 2012.

Reference Source

Ghurye J, Rhie A, Walenz BP, et al.: Integrating Hi-C Links with Assembly Graphs for Chromosome-Scale Assembly. PLoS Comput Biol. 2019; 15(8): e1007273.

PubMed Abstract | Publisher Full Text | Free Full Text
Guan D, McCarthy SA, Wood J, et al.: Identifying and Removing Haplotypic Duplication in Primary Genome Assemblies. Bioinformatics. 2020; 36(9): 2896-98.

PubMed Abstract | Publisher Full Text | Free Full Text

Howe K, Chow W, Collins J, et al.: Significantly Improving the Quality of Genome Assemblies through Curation. GigaScience. 2021; 10(1): giaa153. PubMed Abstract | Publisher Full Text | Free Full Text

Kerpedjiev P, Abdennur N, Lekschas F, et al.: HiGlass: Web-Based Visual Exploration and Analysis of Genome Interaction Maps. Genome Biol. 2018; 19(1): 125. PubMed Abstract | Publisher Full Text | Free Full Text

Majerus MEN: Inheritance of Three Common Forms of Acronicta Aceris (L.)(Lepidoptera: Noctuidae). In Proceedings and Transactions of the British Entomological and Natural History Society. 1986

Reference Source

Manni M, Berkeley MR, Seppey M, et al.: BUSCo Update: Novel and Streamlined Workflows along with Broader and Deeper Phylogenetic Coverage for Scoring of Eukaryotic, Prokaryotic, and Viral Genomes. Mol 
Biol Evol. 2021; 38(10): 4647-54.

PubMed Abstract | Publisher Full Text | Free Full Text

Rao SSP, Huntley MH, Durand NC, et al.: A 3D Map of the Human Genome at Kilobase Resolution Reveals Principles of Chromatin Looping. Cell. 2014;

159(7): $1665-80$.

PubMed Abstract | Publisher Full Text | Free Full Text
Uliano-Silva M, Nunes JGF, Krasheninnikova K, et al.: marcelauliano/MitoHiFi: mitohifi_v2.0. Zenodo. 2021

Publisher Full Text

Waring P, Townsend M, Lewington R: Field Guide to the Moths of Great Britain and Ireland. British Wildlife Publishing, Hampshire. 2003. Reference Source 


\section{Open Peer Review}

\section{Current Peer Review Status:}

\section{Version 1}

Reviewer Report 24 December 2021

https://doi.org/10.21956/wellcomeopenres.19184.r47540

(C) 2021 Djikeng A. This is an open access peer review report distributed under the terms of the Creative Commons Attribution License, which permits unrestricted use, distribution, and reproduction in any medium, provided the original work is properly cited.

\section{Appolinaire Djikeng}

Centre for Tropical Livestock Genetics and Health (CTLGH), Roslin Institute, University of Edinburgh, Edinburgh, UK

The Wellcome Open Research paper by Boyes et al. is a genome sequencing report of the sycamore, Acronicta aceris, found the Southeast and central parts of England.

The methods and strategies for sample collection (a female A. aceris), processing (dissection), preparation and quality control of DNA and sequencing are clearly described and appropriately referenced. Three complementary approaches including the PacificBiosciences SEQUEL II, the 10x genomics Illumina and $\mathrm{Hi}-\mathrm{C}$ Illumina were used to generate high quality genome sequencing data. The data generated was further analysed very convincingly using the latest strategies for genome assembly, construction of 32 chromosomal pseudomolecules and assembly of sex chromosomes. The results are of excellent quality and add a lot more to the much-needed global genomic resources that will definitely support subsequent studies in population dynamics, distribution and the genomic changes that underpin adaptation to some environmental cues.

I fully support this publication but wish (in addition to the comments/inputs already provided by reviewer 1) to make the following suggestions for future consideration:

1. A stronger justification for sequencing this species and the added value to the already existing other genomic resources from closely related species.

2. Under the section on data availability, it would be beneficial to steer how the data will be annotated and if there will be need to generate RNAseq data for better annotation. Additionally, I could also be useful to mention if or how within the DToL initiative to there are plans to generate additional genomic resources to increase the usefulness of this dataset. For example, these genomic resources could be further analysed and discussed in the context of understanding the genomic differences amongst devastating pests (from outside the UK).

Is the rationale for creating the dataset(s) clearly described? 
Yes

Are the protocols appropriate and is the work technically sound?

Yes

Are sufficient details of methods and materials provided to allow replication by others? Yes

Are the datasets clearly presented in a useable and accessible format?

Yes

Competing Interests: No competing interests were disclosed.

Reviewer Expertise: Tropical livestock genomics and genetic improvement

I confirm that I have read this submission and believe that I have an appropriate level of expertise to confirm that it is of an acceptable scientific standard.

Reviewer Report 06 December 2021

https://doi.org/10.21956/wellcomeopenres.19184.r47285

(C) 2021 Singh K. This is an open access peer review report distributed under the terms of the Creative Commons Attribution License, which permits unrestricted use, distribution, and reproduction in any medium, provided the original work is properly cited.

\section{Kumar Saurabh Singh}

${ }^{1}$ Bioinformatics Group, Wageningen University, Wageningen, The Netherlands

2 Plant-Microbe Interaction Group, Utrecht University, Utrecht, The Netherlands

3 College of Life and Environmental Sciences, University of Exeter, Cornwall Campus, Cornwall, UK

Boyes et al. have sequenced a genome of Acronicta aceris, a Lepidopteran from Noctuidae family. They have assembled the genome using PacBio HiFi long-read sequencing technology and scaffolded the genome using Illumina Novaseq based on short-read data obtained from chromosome conformation capture $\mathrm{Hi}-\mathrm{C}$ technology. To polish the genome high-coverage $10 \mathrm{X}$ genomics short-read data was also generated. Based on the adopted methodologies for genome sequencing, assembly, and quality checks, I am very much convinced that the genome is of very high quality (Contig N50:15.4 Mb; Scaffold N50: 16.1 Mb; Longest contig: 19 Mb; BUSCO C: 99\%) with 32 binned chromosomes including the sex chromosomes and a mitochondrial genome. However, I have some minor comments which might improve the quality of this genome note:

1. I think a few lines of the motivation behind the sequencing of this moth is missing in the background. Particularly, I would like to see why it is important to sequence the genome of this moth and what value it will bring to the overall Lepidopteran genomics.

2. Authors have performed one round of polishing using 10X short-read data, but they have not given any information about what proportion of variants they have observed before and 
after polishing. This will further add knowledge to the quality of the long-reads and to the overall assembly quality.

3. I think it would be useful to the readers if the authors add an estimate of the base-level accuracy (QV) for the assembly. QV values are useful for downstream applications like population or functional genetics. This stat could be easily calculated using Merqury (Rhie et al. Genome Biol 21, $\left.245\left(2020^{1}\right)\right)$

\section{References}

1. Rhie A, Walenz B, Koren S, Phillippy A: Merqury: reference-free quality, completeness, and phasing assessment for genome assemblies. Genome Biology. 2020; 21 (1). Publisher Full Text

Is the rationale for creating the dataset(s) clearly described?

Yes

Are the protocols appropriate and is the work technically sound?

Yes

Are sufficient details of methods and materials provided to allow replication by others? Yes

Are the datasets clearly presented in a useable and accessible format? Yes

Competing Interests: No competing interests were disclosed.

Reviewer Expertise: Bioinformatics; Genome informatics; Integrative omics;

I confirm that I have read this submission and believe that I have an appropriate level of expertise to confirm that it is of an acceptable scientific standard. 